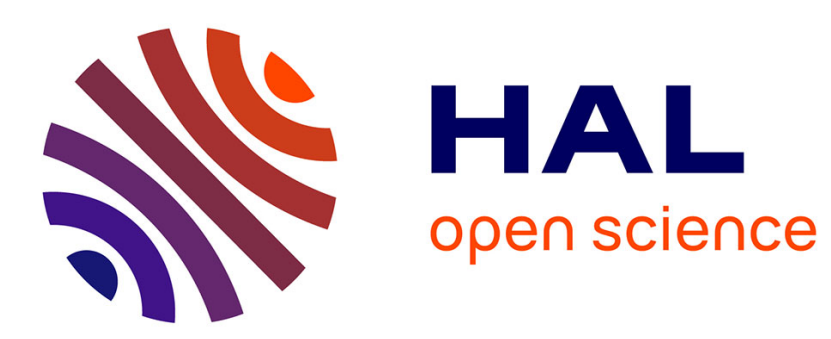

\title{
A Metric Approach to nD Images Edge Detection With Clifford Algebras
}

Thomas Batard, Christophe Saint-Jean, Michel Berthier

\section{To cite this version:}

Thomas Batard, Christophe Saint-Jean, Michel Berthier. A Metric Approach to nD Images Edge Detection With Clifford Algebras. Journal of Mathematical Imaging and Vision, 2009, 33 (3), pp.296312. 10.1007/s10851-008-0115-0 . hal-00267629v2

\section{HAL Id: hal-00267629 \\ https://hal.science/hal-00267629v2}

Submitted on 17 Sep 2008

HAL is a multi-disciplinary open access archive for the deposit and dissemination of scientific research documents, whether they are published or not. The documents may come from teaching and research institutions in France or abroad, or from public or private research centers.
L'archive ouverte pluridisciplinaire HAL, est destinée au dépôt et à la diffusion de documents scientifiques de niveau recherche, publiés ou non, émanant des établissements d'enseignement et de recherche français ou étrangers, des laboratoires publics ou privés. 


\title{
A Metric Approach to nD Images Edge Detection With Clifford Algebras
}

\author{
Thomas Batard - Christophe Saint-Jean - Michel Berthier
}

\begin{abstract}
The aim of this paper is to perform edge detection in color-infrared images from the point of view of Clifford algebras. The main idea is that such an image can be seen as a section of a Clifford bundle associated to the $R G B T$-space (Red, Green, Blue, Temperature) of acquisition. Dealing with geometric calculus and covariant derivatives of appropriate sections with respect to well-chosen connections allows to get various color and temperature information needed for the segmentation. We show in particular how to recover the first fundamental form of the image embedded in a LSHT-space (Luminance, Saturation, Hue, Temperature) equipped with a metric tensor. We propose applications to color edge detection with some constraints on colors and to edge detection in color-infrared images with constraints on both colors and temperature. Other applications related to different choices of connections, sections and embedding spaces for $\mathrm{nD}$ images may be considered from this general theoretical framework.
\end{abstract}

Keywords Edge detection - Clifford algebras bundle . Geometric calculus · Color · Infrared · Di Zenzo gradient

\footnotetext{
T. Batard, C. Saint-Jean, M. Berthier

Laboratoire Mathématiques, Image et Applications

Université de La Rochelle

Avenue Michel Crépeau, 17042 La Rochelle Cedex, France

T. Batard (corresponding author)

Tel.: +33(0) 546-458-629

Fax: +33(0) 546-458-240

E-mail: thomas.batard01@univ-lr.fr

C. Saint-Jean

E-mail: christophe.saint-jean@univ-lr.fr

M. Berthier

E-mail: michel.berthier@univ-lr.fr
}

\section{Introduction}

Clifford algebras appear to be a powerful tool in a wide range of applications to computer sciences, see [28] for examples. In particular, the approach of Sangwine \& al. of color images segmentation with quaternions [25], [12] can be considered from this viewpoint since $\mathbb{H}$ (the algebra of quaternions) is the Clifford algebra $\mathbb{R}_{0,2}$ and $\mathbb{H}^{1}$ (the group of unit quaternions) is the spinor group Spin(3). Sangwine's idea is to associate a pure imaginary quaternion to each color of the RGB cube and then make geometric transformations on colors using the product of $\mathbb{H}$ to compute some kind of gradient.

Working in the framework of Clifford algebras has several advantages:

- If we consider a color as a vector of the algebra $\mathbb{R}_{3,0}$ we can use the richness of the structure of this latter. In fact $\mathbb{R}_{3,0}$ is of dimension 8 over $\mathbb{R}$ and contains elements of different degrees (scalars, vectors, bivectors and a pseudoscalar) that carry different information.

- We can benefit from the efficiency of the calculus based on the geometric product when dealing with general images with values in $\mathbb{R}^{n}$. As it is well known, geometric transformations can be encoded without coordinates by algebraic formula using for example spinors. We will consider in the sequel the example of colorinfrared images where $n=4$.

- A Clifford algebra is defined with respect to a chosen metric of a vector space. Conjointly with the two preceding points, it's an asset when dealing with metric approach of edge detection. In particular, when the metric of the ambient space varies with the point, the Clifford algebras bundle setting arises quite naturally.

The metric approach we adopt in this paper was introduced by Di Zenzo [10], who considered implicitely a $n$-channels image as an embedded two-dimensional 
surface in the Euclidean space $\mathbb{R}^{n+2}$. A measure of the "edge strength" and the direction wherein it is highest at each point are defined. This is done by computing respectively the highest eigenvalue of the first fundamental form of the surface and the corresponding eigenvector. Using this method, Cumani [9] gives an explicit definition of an edge point, that is a point where the first directional derivative of the highest eigenvalue in the direction given by the highest eigenvector has a transversal zero-crossing. However, when $n>1$, the smallest eigenvalue is no more constant; that's why Sapiro [26] suggests as a measure of the "edge strength" the square root of the difference of the two eigenvalues.

The idea of embedding the surface representing an image in a space endowed with a non-Euclidean metric is proposed in [27], and is applied for color edge detection in [22]. In this latter, the author describes colors with hyperbolic coordinates and endows $\mathbb{R}^{5}$ with a corresponding metric.

It is our purpose to start from this general definition of a $\mathrm{nD}$ image and to develop a general approach of edge detection in color-infrared images based on metric information. Indeed, we define an image as a section of a fiber bundle. The fibers are Clifford algebras related to the acquisition space $R G B T$ of the color-infrared image endowed with a metric that may vary with the base point. We show that it is possible, staying in the acquisition space, to recover the metric data of the image

$$
\varphi:(x, y) \longmapsto(x, y, l, s, h, t)
$$

embedded in a $L S H T$-space $(l, s, h, t$, denotes respectively the luminance, the saturation, the hue and the temperature) equipped with a metric tensor. This is done in both continuous and discrete cases by considering two sections of the Clifford bundle and their covariant derivatives with respect to a well chosen connection.

We may insist on the following facts:

- We never compute explicitely the derivatives of luminance, saturation, hue and temperature whereas the approach based on surfaces does.

- The information contained in the various channels (for example color and temperature in application 4.3) is mixed to adjust the coefficients of the metric of the embedding space. That is why the process we propose can't be achieved with marginal approaches.

This paper is organized as follows. Sect. 2 is mainly devoted to basic notions and results on Clifford algebras and spinor groups. We show also how to derive the transition formulas from $R G B T$ to $L S H T$ with geometric calculus. In Sect. 3, we first compute the coefficients of the first fundamental form of a color-infrared image defined as a two-dimensional surface embedded in a $L S H T$-space equipped with a metric tensor. Then, we show how to interpret such an image as a section of a Clifford algebras bundle. The rest of the section is devoted to the continuous and discrete computations of the coefficients of the first fundamental form as explained above. In Sect. 4, we propose three applications: a comparison with the Di Zenzo's method, a detection of transitions between colors of a given hue interval with respect to their saturation levels and an edge detection in a color-infrared image with constraints on color and temperature of objects. We discuss also other applications, related to different choices of connections and sections, to show the genericity of the proposed method. In particular, segmentations of shadows and highlights are briefly investigated.

\section{Clifford algebras and color-infrared spaces}

\subsection{Clifford algebras}

The aim of this section is to give basic definitions and results concerning Clifford algebras and spinor groups. We refer the reader to [8], [21], [19], [20], [3], [24], [23], [7] for further details on history, definitions, results and applications.

Let $V$ be a vector space of finite dimension $n$ over $\mathbb{R}$ equipped with a quadratic form $Q$. We are looking for an algebra $C l(V, Q)$ that contains $V$ and in which the following relation holds for all $v$ in $V$ :

$$
v^{2}=Q(v) \cdot 1
$$

This implies in particular that for all $u$ and $v$ in $V$ :

$$
u v+v u=2 B(u, v) \cdot 1
$$

where $B$ denotes the symmetric bilinear form corresponding to $Q$. We can decompose the product $u v$ into

$$
u v=B(u, v) \cdot 1+u \wedge v
$$

by defining

$$
u \wedge v=\frac{1}{2}(u v-v u) .
$$

This shows that $C l(V, Q)$ contains with scalars and vectors other elements called bivectors and generally multivectors which appear to generalize the notion of vector. It is possible to add and multiply all the elements, the multiplication being not commutative.

As an example, let us mention that the Clifford algebra of $\mathbb{R}^{2}$ with the quadratic form

$$
Q\left(u_{1}, u_{2}\right)=-u_{1}^{2}-u_{2}^{2}
$$

is isomorphic to the algebra of quaternions: An isomorphism is given by sending respectively $1, e_{1}, e_{2}$ and $e_{1} e_{2}$ to $1, i, j$ and $k$ ). 
Formally speaking, the Clifford algebra $C l(V, Q)$ is the solution of the following universal problem. We search a couple $\left(C l(V, Q), i_{Q}\right)$ where $C l(V, Q)$ is an $\mathbb{R}$-algebra and $i_{Q}: V \longrightarrow C l(V, Q)$ is $\mathbb{R}$-linear satisfying:

$$
\left(i_{Q}(v)\right)^{2}=Q(v) \cdot 1
$$

for all $v$ in $V(1$ denotes the unit of $C l(V, Q))$ such that for each $\mathbb{R}$-algebra $A$ and each $\mathbb{R}$-linear map $f: V \longrightarrow$ $A$ with

$$
(f(v))^{2}=Q(v) \cdot 1
$$

for all $v$ in $V(1$ denotes the unit of $A)$, then there exists a unique morphism

$$
g: C l(V, Q) \longrightarrow A
$$

of $\mathbb{R}$-algebras such that $f=g \circ i_{Q}$.

The solution is unique up to isomorphisms and is given as the (non commutative) quotient

$$
T(V) /(v \otimes v-Q(v) .1)
$$

of the tensor algebra of $V$ by the ideal generated by $v \otimes v-Q(v) .1$, where $v$ belongs to $V$ (see [24] for a proof).

It is well known that there exists a unique anti-automorphism $t$ on $C l(V, Q)$ such that

$$
t\left(i_{Q}(v)\right)=i_{Q}(v)
$$

for all $v$ in $V$. It is called reversion and usually denoted by $x \longmapsto x^{\dagger}, x$ in $C l(V, Q)$. In the same way there exists a unique automorphism $\alpha$ on $C l(V, Q)$ such that

$$
\alpha\left(i_{Q}(v)\right)=-i_{Q}(v)
$$

for all $v$ in $V$. In the rest of this paper we write $v$ for $i_{Q}(v)$ (according to the fact that $i_{Q}$ embeds $V$ in $C l(V, Q))$.

As a vector space $C l(V, Q)$ is of dimension $2^{n}$ on $\mathbb{R}$ and a basis is given by the set

$$
\left\{e_{i_{1}} e_{i_{2}} \cdots e_{i_{k}}, \quad i_{1}<i_{2}<\ldots<i_{k}, \quad k \in\{1, \ldots, n\}\right\}
$$

and the unit 1 . An element of degree $k$

$$
\sum_{i_{1}<\cdots<i_{k}} \alpha_{i_{1} \ldots i_{k}} e_{i_{1}} e_{i_{2}} \cdots e_{i_{k}}
$$

is called a $k$-vector. A 0 -vector is a scalar and $e_{1} e_{2} \cdots e_{n}$ is called the pseudoscalar. We will denote $\langle x\rangle_{k}$ the component of degree $k$ of an element $x$ of $C l(V, Q)$.

The inner product of $x_{r}$ of degree $r$ and $y_{s}$ of degree $s$ is defined by

$$
x_{r} \cdot y_{s}=\left\langle x_{r} y_{s}\right\rangle_{|r-s|}
$$

if $r$ and $s$ are positive and by

$$
x_{r} \cdot y_{s}=0
$$

otherwise.
The outer product of $x_{r}$ of degree $r$ and $y_{s}$ of degree $s$ is defined by

$$
x_{r} \wedge y_{s}=\left\langle x_{r} y_{s}\right\rangle_{r+s}
$$

These products extend by linearity on $C l(V, Q)$. Clearly, if $a$ and $b$ are vectors of $V$, then the inner product of $a$ and $b$ coincides with the scalar product defined by $Q$. When it is defined (for example when $x$ is a versor and $Q$ is positive) we denote

$$
\|x\|=\sqrt{x x^{\dagger}}
$$

and say that $x$ is a unit if $x x^{\dagger}= \pm 1$.

In the following, we deal in particular with the Clifford algebra of the Euclidean $\mathbb{R}^{n}$ denoted by $\mathbb{R}_{n, 0}$. $\mathbb{R}_{n, 0}^{k}$ is the subspace of elements of degree $k$ and $\mathbb{R}_{n, 0}^{*}$ is the group of elements that admit an inverse in $\mathbb{R}_{n, 0}$.

Let $a$ be a vector in $\mathbb{R}_{n, 0}$ and $B$ be the $k$-vector $a_{1} \wedge$ $a_{2} \wedge \cdots \wedge a_{k}$, then the orthogonal projection of $a$ on the $k$-plane generated by the $a_{i}$ 's is the vector

$$
P_{B}(a)=(a \cdot B) B^{-1}
$$

The vector

$$
a-(a \cdot B) B^{-1}=(a \wedge B) B^{-1}
$$

is called the rejection of $a$ on $B$.

\subsection{The spinor group $\operatorname{Spin}(n)$}

It is defined by

$$
\operatorname{Spin}(n)=\left\{\prod_{i=1}^{2 k} a_{i}, a_{i} \in \mathbb{R}_{n, 0}^{1},\left\|a_{i}\right\|=1\right\}
$$

or equivalently

$$
\begin{gathered}
\operatorname{Spin}(n)=\left\{x \in \mathbb{R}_{n, 0}, \alpha(x)=x, x x^{\dagger}=1,\right. \\
\left.x v x^{-1} \in \mathbb{R}_{n, 0}^{1} \forall v \in \mathbb{R}_{n, 0}^{1}\right\}
\end{gathered}
$$

It is well known that $\operatorname{Spin}(n)$ is a connected compact Lie group that universally covers $\mathrm{SO}(n)(n \geq 3)$. One can verify that $\operatorname{Spin}(3)$ is the group

$$
\left\{a 1+b e_{1} e_{2}+c e_{2} e_{3}+d e_{3} e_{1}, a^{2}+b^{2}+c^{2}+d^{2}=1\right\}
$$

and is isomorphic to the group $\mathbb{H}^{1}$ of unit quaternions. It is also a classical result that $\operatorname{Spin}(4)$ is isomorphic to $\operatorname{Spin}(3) \times \operatorname{Spin}(3)$ (see [23] for more information on spinors in $\mathbb{R}^{3}$ and $\left.\mathbb{R}^{4}\right)$. 
The Lie algebra of $\operatorname{Spin}(n)$ is $\mathbb{R}_{n, 0}^{2}$ with Lie bracket

$$
A \times B=A B-B A
$$

As the exponential map from its Lie algebra to $\operatorname{Spin}(n)$ is onto (see [18] for a proof), every spinor can be written as

$$
S=\sum_{i=0}^{\infty} \frac{1}{i !} A^{i}
$$

for some bivector $A$.

From Hestenes and Sobczyk [21], we know that every $A$ in $\mathbb{R}_{n, 0}^{2}$ can be written as

$$
A=A_{1}+A_{2}+\cdots+A_{m}
$$

where $m \leq n / 2$ and

$$
A_{j}=\left\|A_{j}\right\| a_{j} b_{j}, \quad j \in\{1, \ldots, m\}
$$

with

$$
\left\{a_{1}, \ldots, a_{m}, b_{1}, \ldots, b_{m}\right\}
$$

a set of orthonormal vectors. Thus

$$
A_{j} A_{k}=A_{k} A_{j}=A_{k} \wedge A_{j}
$$

whenever $j \neq k$ and

$$
A_{k}^{2}=-\left\|A_{k}\right\|^{2}<0
$$

This means that the planes encoded by $A_{k}$ and $A_{j}$ are orthogonal and implies that

$$
e^{A_{1}+A_{2}+\cdots+A_{m}}=e^{A_{\sigma(1)}} e^{A_{\sigma(2)}} \ldots e^{A_{\sigma(m)}}
$$

for all $\sigma$ in the permutation group $\mathfrak{S}(m)$. Actually, as $A_{k}^{2}$ is negative we have

$$
e^{A_{i}}=\cos \left(\left\|A_{i}\right\|\right)+\sin \left(\left\|A_{i}\right\|\right) \frac{A_{i}}{\left\|A_{i}\right\|}
$$

The corresponding rotation

$$
R_{i}: x \longmapsto e^{-A_{i}} x e^{A_{i}}
$$

acts in the oriented plane defined by $A_{i}$ as a plane rotation of angle $2\left\|A_{i}\right\|$. The vectors orthogonal to $A_{i}$ are invariant under $R_{i}$.

It then appears that any element $R$ of $\mathrm{SO}(n)$ is a composition of commuting simple rotations, in the sense that they have only one invariant plane. The vectors left invariant by $R$ are those of the orthogonal subspace to $A$. If $m=n / 2$ this latter is trivial. The previous decomposition is not unique if $\left\|A_{k}\right\|=\left\|A_{j}\right\|$ for some $j$ and $k$ with $j \neq k$. In this case infinitely many planes are left invariant by $R$.

\subsection{Color-infrared spaces}

As mentioned before, Sangwine's approach of edge detection in color images relies on the fact that $\mathbb{H}_{0}$ (the set of pure imaginary quaternions) is isomorphic to $\mathbb{R}^{3}$ equipped with an action of $\mathbb{H}^{1}$. In the same way, a color image can be treated as an application from $\mathbb{R}^{2}$ to $\mathbb{R}^{3}$, this latter being embedded in $\mathbb{R}_{3,0}$ and equipped with an action of $\operatorname{Spin}(3)$. It is natural to extend this approach to $\mathrm{nD}$ images replacing $\mathbb{R}^{3}$ by $\mathbb{R}^{n}$ and $\mathbb{R}_{3,0}$ by $\mathbb{R}_{n, 0}$. We focus here on color-infrared images.

Beside RGB color space we consider HSL color space defined as follows. We set first

$$
\left(\begin{array}{l}
Y \\
C_{1} \\
C_{2}
\end{array}\right)=\left(\begin{array}{ccc}
1 / 3 & 1 / 3 & 1 / 3 \\
1 & -1 / 2 & -1 / 2 \\
0 & -\sqrt{3} / 2 & \sqrt{3} / 2
\end{array}\right)\left(\begin{array}{l}
r \\
g \\
b
\end{array}\right)
$$

Then the luminance $l$, the saturation $s$ and the hue $h$ are respectively given by

$$
\begin{gathered}
l=Y \\
s=\sqrt{C_{1}^{2}+C_{2}^{2}} \\
h=\left\{\begin{array}{cc}
\arccos \left(C_{2} / s\right) & \text { if } C_{2}>0 \\
2 \pi-\arccos \left(C_{2} / s\right) & \text { otherwise }
\end{array}\right.
\end{gathered}
$$

As it is well known, color spaces based on luminance (value), saturation and hue are more suitable to perception [13].

Let us denote $\mathcal{C} \mathcal{T}$ the Clifford algebra of $\left(\mathbb{R}^{4}, Q\right)$ with $Q$ the positive definite quadratic form given by

$$
\left(\begin{array}{cccc}
\beta / 3 & 0 & 0 & 0 \\
0 & \beta / 3 & 0 & 0 \\
0 & 0 & \beta / 3 & 0 \\
0 & 0 & 0 & \delta
\end{array}\right)
$$

Thus $e_{1}^{2}=e_{2}^{2}=e_{3}^{2}=\beta / 3$ and $e_{4}^{2}=\delta$. Given a colorinfrared vector $a=r(a) e_{1}+g(a) e_{2}+b(a) e_{3}+t(a) e_{4}$, its color component is given by

$$
\begin{aligned}
c(a) & =r(a) e_{1}+g(a) e_{2}+b(a) e_{3} \\
& =a \cdot\left(e_{1} e_{2} e_{3}\right)\left(e_{1} e_{2} e_{3}\right)^{-1}
\end{aligned}
$$

Let us denote

$$
\mu=\frac{e_{1}+e_{2}+e_{3}}{\sqrt{\beta}}
$$

the unit vector generating the achromatic axis, and $v(a)$ the rejection of $c(a)$ on $\mu$, called the chrominance vector of $a$. Simple computations show that the luminance $l(a)$, the saturation $s(a)$ and the hue $h(a)$ of $a$ can be written

$$
\begin{aligned}
l(a) & =\frac{1}{\sqrt{\beta}}\left\|(a \cdot \mu) \mu^{-1}\right\| \\
& =\frac{1}{\sqrt{\beta}} \sqrt{\left((a \cdot \mu) \mu^{-1}\right)^{2}}
\end{aligned}
$$




$$
\begin{gathered}
s(a)=\frac{3}{\sqrt{2 \beta}}\left\|(c(a) \wedge \mu) \mu^{-1}\right\| \\
=\frac{3}{\sqrt{2 \beta}} \sqrt{\left((c(a) \wedge \mu) \mu^{-1}\right)^{2}} \\
h(a)=2 \pi+\operatorname{sign}(g(a)-b(a)) \arccos \left(\frac{v(a)}{\|v(a)\|} \cdot \rho\right)
\end{gathered}
$$

with $\rho$ the unit chrominance vector of the red color and $h(a)$ defined modulo $2 \pi$. In other words, $h(a)$ is the oriented angle from $\rho$ to $v(a)$.

Moreover, the dual of the achromatic axis in the vector space generated by $\left(e_{1}, e_{2}, e_{3}\right)$ is a plane, called the chrominance plane, generated by the bivector

$$
e_{1} e_{2}-e_{1} e_{3}+e_{2} e_{3}
$$

and we have

$$
v(a)=a \cdot\left(e_{1} e_{2}-e_{1} e_{3}+e_{2} e_{3}\right)\left(e_{1} e_{2}-e_{1} e_{3}+e_{2} e_{3}\right)^{-1}
$$

The chrominance vector of $a$ is therefore the orthogonal projection of $a$ on the chrominance plane.

\section{Edge detection in color-infrared images}

3.1 First fundamental form of a surface and edge detectors

We recall in this subsection how to define an edge detector using metric information given by the first fundamental form (see [10], [9], [26] and [27], [29] for related works on edge-preserving denoising). For this we consider a color-infrared image as a $C^{k}$ map, $k \geq 1$,

$$
\varphi:(x, y) \longmapsto(x, y, l(x, y), s(x, y), h(x, y), t(x, y))
$$

from a rectangle $D$ to $\mathbb{R}^{6}$. In the following $q$ denotes a point in $D$ with image $p=\varphi(q)$ under the map $\varphi$. Note that we consider the hue $h$ with values in the universal cover $\mathbb{R}$ of $\mathbb{R} / 2 \pi \mathbb{Z}$. For a coherent definition of $\varphi$, we take $h=0$ when $s=0$. As we will see below, this has no consequences on the edge detection.

To take into account the fact that the hue is irrelevant for small values of the saturation, we introduce following Carron [5] the function

$$
f(s)=\frac{1}{\pi}\left(\frac{\pi}{2}+\arctan \left(\beta\left(s-S_{0}\right)\right)\right) .
$$

$f$ measures the relevance of hue with respect to saturation level. The shape of $f$ is controlled by two parameters $S_{0}$ and $\beta$. $S_{0}$ is the saturation level corresponding to the medium relevance of hue: $f\left(S_{0}\right)=0.5$. The parameter $\beta$ is the slope of the function around $S_{0}$. In the sequel we choose $S_{0}=50$ and $\beta=0.07$. The reader may find in [6] similar constructions. We consider also the domain

$$
\Omega(p)=\left\{(x, y),\left\|(x, y)-\varphi^{-1}(p)\right\|_{\infty} \leq 1\right\}
$$

and set

$$
\xi(p)=\exp \left(\frac{1}{4} \int_{\Omega(p)} \ln (f \circ s(x, y)) d x d y\right)
$$

if $s(u, v) \neq 0$ for all $(u, v)$ in $\Omega(p)$ and $\xi(p)=0$ otherwise.

Next, we endow $\mathbb{R}^{6}$ with the following metric

$$
\left(\begin{array}{ll}
1 & 0 \\
0 & 1
\end{array}\right) \oplus\left(\begin{array}{cccc}
\lambda(p) & 0 & 0 & 0 \\
0 & \lambda(p) & 0 & 0 \\
0 & 0 & \kappa(p) \xi(p) & 0 \\
0 & 0 & 0 & \eta(p)
\end{array}\right)
$$

where $\lambda, \kappa$ and $\eta$ are positive functions. Strictly speaking, as $\xi$ can vanish, this metric is not Riemannian. However the metric induced on the surface $\varphi(D)$ is Riemannian: It is the first fundamental form of $\varphi(D)$, usually denoted by

$$
\mathrm{I}(p)=\left(\begin{array}{cc}
E(p) & F(p) \\
F(p) & G(p)
\end{array}\right)
$$

The coefficients $E, F$ and $G$ are given by

$$
\begin{gathered}
E(p)=1+\lambda(p) l_{x}^{2}(p)+\lambda(p) s_{x}^{2}(p) \\
+\kappa(p) \xi(p) h_{x}^{2}(p)+\eta(p) t_{x}^{2}(p) \\
F(p)=\lambda(p) l_{x}(p) l_{y}(p)+\lambda(p) s_{x}(p) s_{y}(p) \\
+\kappa(p) \xi(p) h_{x}(p) h_{y}(p)+\eta(p) t_{x}(p) t_{y}(p) \\
G(p)=1+\lambda(p) l_{y}^{2}(p)+\lambda(p) s_{y}^{2}(p) \\
+\kappa(p) \xi(p) h_{y}^{2}(p)+\eta(p) t_{y}^{2}(p)
\end{gathered}
$$

We denote $\theta_{+}(p)$ and $\theta_{-}(p), \theta_{+}(p) \geq \theta_{-}(p)$, the two eigenvalues of $\mathrm{I}(p)$ and $\Theta_{+}(p), \Theta_{-}(p)$ the corresponding eigenvectors. The edge detector is then given by

$$
\varpi(p)=\sqrt{\theta_{+}(p)-\theta_{-}(p)}
$$

More precisely, we say that $q$ in $D$ is an edge point if one of the following conditions holds:

1. The function $\varpi$ has a local maximum at $\varphi(q)$ in the direction given by $\Theta_{+}(\varphi(q))$;

2. $\Theta_{+}(\varphi(q))>1$ and $q$ is an endpoint of a curve of points satisfying 1 . 


\subsection{Clifford bundle and color-infrared image}

We explain how to consider a color/infrared image as a section of a Clifford bundle. First of all, let us recall some definitions.

A vector bundle of rank $n$ over a surface $S$ (or more generally over a manifold) consists of a family $\left\{E_{p}\right\}_{p \in S}$ of $n$-dimensional vector spaces parametrized by $S$ together with a differentiable manifold structure on

$$
E=\bigcup_{p \in S} E_{p}
$$

that satisfy the following conditions.

- The projection map $\pi: E \longrightarrow S$ taking $E_{p}$ to $p$ is differentiable $\left(E_{p}\right.$ is called the fiber above $\left.p\right)$.

- For every $p$ in $S$, there exists an open set $U$ in $S$ containing $p$ and a diffeomorphism

$$
\varphi_{U}: \pi^{-1}(U) \longrightarrow U \times \mathbb{R}^{n}
$$

taking the vector space $E_{p}$ isomorphically onto $\{p\} \times$ $\mathbb{R}^{n}$. The diffeomorphism $\varphi_{U}$ is called of trivialization of $E$ over $U$, and $\mathbb{R}^{n}$ the typical fiber. If moreover there exists a trivialization over $S$, we say that $E$ is a trivial vector bundle. Two examples of major importance are the tangent and cotangent bundles, $T S$ and $T^{*} S$, corresponding respectively to $E_{p}=T_{p} S$ (the tangent space to $S$ at $p$ ) and $E_{p}=T_{p}^{*} S$ (the cotangent space to $S$ at $p)$. We denote $\Gamma(S, E)$ the module of sections of $E$ over $S$.

Vector bundles are particular cases of fiber bundles, where we only require the fibers to be topological spaces. In the sequel, we will sometimes use the term fiber bundle when talking about the Clifford bundle we consider.

A section $\sigma$ of the vector bundle $E$ over $S$ is a differentiable map

$$
\sigma: S \longrightarrow E
$$

such that $\sigma(p)$ belongs to $E_{p}$ for all $p$ in $S$. It is well known that sections of $T S$, resp. $T^{*} S$, correspond to vector fields, resp. 1-forms, on $S$.

One more ingredient that is used in the sequel is the notion of connection (see [14]). A connection $\nabla$ on a fiber bundle $E$ is an operator taking sections $\sigma$ of $E$ into $E$-valued 1 -forms $\nabla \sigma$ such that the Leibniz rule holds; if $f$ is a function, then

$$
\nabla(f \sigma)=f \nabla \sigma+\sigma \otimes d f
$$

A connection is essentially a way of differentiating sections. To any connection we associate covariant derivatives that generalize to vector bundles the notion of directional derivatives on vector-valued functions.
Lots of computations we make in the next subsection necessitate to deal with tensor products, the definition and properties of which can be found in [1] and [24].

Let \|\|$_{2}$ be the Euclidean norm on $\mathbb{R}^{n}$. Keeping the notations of 3.1, we associate to each point $q$ of $D$ the Clifford algebra $\mathcal{C} \mathcal{T}(q)$ of the four-dimensional vector space containing the $R G B T$-space endowed with the metric $Q(q)$

$$
\left(\begin{array}{cccc}
\lambda(q) / 3 & 0 & 0 & 0 \\
0 & \lambda(q) / 3 & 0 & 0 \\
0 & 0 & \lambda(q) / 3 & 0 \\
0 & 0 & 0 & \eta(q)
\end{array}\right)
$$

(compare with Sect. $2.3: \beta$ is replaced by $\lambda(q)$ and $\delta$ is replaced by $\eta(q))$. Let $\mathcal{C} \mathcal{T}(D)$ be the disjoint union of $\mathcal{C} \mathcal{T}(q)$ for $q$ in $D$.

Proposition $1 \mathcal{C} \mathcal{T}(D)$ with the projection

$$
\pi: \mathcal{C} \mathcal{T}(D) \longrightarrow D
$$

that maps $\zeta \in \mathcal{C} \mathcal{T}(q)$ to $q$ is a trivial vector bundle $(\mathcal{C} \mathcal{T}(D), D, \pi)$ with typical fiber $C l\left(\mathbb{R}^{4},\|\|_{2}\right)$.

Proof We have to show that there exists a diffeomorphism $\Phi$ from $\pi^{-1}(D)$ onto $D \times C l\left(\mathbb{R}^{4},\|\|_{2}\right)$ such that $\Phi \circ p_{1}=\pi$ where $p_{1}$ denotes the projection on the first factor. As $\mathcal{C} \mathcal{T}(q)$ is isomorphic to $C l\left(\mathbb{R}^{4},\|\|_{2}\right)$ by some $\Phi_{q}$ for all $q$ in $D$, we can define $\Phi$ by

$$
\Phi:(v \in \mathcal{C} \mathcal{T}(q)) \longmapsto\left(q, \Phi_{q}(v)\right)
$$

It is clearly a diffeomorphism.

In the sequel, we will call such a vector bundle a Clifford algebras bundle or Clifford bundle, since the fibers (those above $D$ and the typical fiber) are endowed with a Clifford algebra structure. In the litterature, when talking about Clifford bundles, we often mean fiber bundles where the fibers are Clifford algebras and the isomorphisms respect the Clifford algebra structure. The situation is different in our case since the isomorphisms are vector space isomorphisms.

A color-infrared image $I$ is now considered as a section

$q \in D \longmapsto r(q) e_{1}(q)+g(q) e_{2}(q)+b(q) e_{3}(q)+t(q) e_{4}(q)$

of $\mathcal{C} \mathcal{T}(D)$

From the fact that $(\mathcal{C} \mathcal{T}(D), D, \pi)$ is trivial we know that any connection on it can be written as

$$
\nabla=d+\omega
$$


for some $\omega$ in $\Gamma\left(D, T^{*} D \otimes \operatorname{End}(\mathcal{C} \mathcal{T}(D)), d\right.$ being the exterior differential [17]. If

$$
X=\left(X_{1}, X_{2}\right)
$$

is a vector field on $D$ and

$$
Y=Y_{0} 1+Y_{1} e_{1}+\ldots+Y_{15} e_{1} e_{2} e_{3} e_{4}
$$

is a section of $\mathcal{C} \mathcal{T}(D)$ then

$$
(\omega(X) Y)_{j}=\sum_{k=0}^{15}\left(\Gamma_{1, k}^{j} X_{1}+\Gamma_{2, k}^{j} X_{2}\right) Y_{k}
$$

so that the connection is entirely determined by the symbols $\Gamma_{i, j}^{k}, i=1,2$ and $j, k=0, \ldots 15$.

In the next paragraph we deal with the following three objects:

i. The connection $\widetilde{\nabla}$ defined by

$$
\Gamma_{i j}^{k}=\left\{\begin{array}{cc}
\frac{\partial_{i} \lambda}{\lambda} & \text { if } k=j \in\{6,7,9\} \\
0 & \text { otherwise }
\end{array}\right.
$$

ii. The section $\psi$ of $(\mathcal{C T}(D), D, \pi)$ given by

$$
\psi=S^{\dagger} I S
$$

with

$$
S=\exp \left[-\frac{h}{2}\left(\frac{e_{1} e_{2}-e_{1} e_{3}+e_{2} e_{3}}{\left\|e_{1} e_{2}-e_{1} e_{3}+e_{2} e_{3}\right\|}\right)\right]
$$

iii. The section $\gamma$ of $(\mathcal{C T}(D), D, \pi)$ given by

$$
\gamma=\frac{v}{\|v\|} \rho
$$

where $\rho$ is the unit chrominance vector of the red color, $v$ is the chrominance vector, $h$ is the hue (see Sect. 2.3) and \|\| means that we take the norm of each fiber $\pi^{-1}(q)$.

\subsection{Computing $E, F, G$ with $\widetilde{\nabla}$}

The main result of this part is that the preceding coefficients $E, F$ and $G$ can be computed using covariant derivatives with respect to $\widetilde{\nabla}$.

\section{Proposition 2 Let}

i. $P_{1}$ (resp. $\left.P_{2}\right)$ be the section of $\operatorname{End}(\mathcal{C T}(D))$ such that $P_{1}(q)$ (resp. $P_{2}(q)$ ) is the orthogonal projection on the plane generated by the luminance and the temperature (resp. on the chrominance plane) in the fiber $\pi^{-1}(q)$;

ii. $d x$ (resp. $d y)$ be the canonical $\mathcal{C} \mathcal{T}(D)$-valued 1 form $d x \otimes 1$ (resp. $d y \otimes 1)$ and $X($ resp. $Y$ ) be the vector field on $D$ of coordinates $(1,0)$ (resp. $(0,1)$ ); iii. $E, F, G$ be the coefficients of the first fundamental form of $\varphi(D)$ (see Sect. 3.1) and $\chi$ be the $\mathcal{C} \mathcal{T}(D)$ valued symmetric tensor of rank 2:

$$
\begin{gathered}
\chi=d x \underline{\otimes} d x+d y \underline{\otimes} d y+P_{1}(\widetilde{\nabla} \psi) P_{1}(\widetilde{\nabla} \psi)+ \\
+\frac{9}{2} P_{2}(\widetilde{\nabla} \psi) \underline{\otimes} P_{2}(\widetilde{\nabla} \psi)-\kappa \xi\left(\gamma^{\dagger} \widetilde{\nabla} \gamma\right) \underline{\otimes}\left(\gamma^{\dagger} \widetilde{\nabla} \gamma\right)
\end{gathered}
$$

then, under the identification of $\mathbb{R}$ and its injection into a Clifford algebra, we have

$$
E=\chi(X \otimes X) \quad F=\chi(X \otimes Y) \quad G=\chi(Y \otimes Y)
$$

(see Sect. 3.1 for the definitions of $\kappa$ and $\xi$ ). In other words, $\chi$ may be viewed as the metric on the surface $\varphi(D)$.

Remarks on the notations. As it is mentioned at the end of the Appendix, the symbol $\underline{\otimes}$ denotes the tensor product over the ring of sections of $\mathcal{C} \mathcal{T}(D)$, whereas the symbol $\otimes$ denotes the tensor product over the ring of $\mathbb{R}$-valued functions on D. $P_{1}(\widetilde{\nabla} \psi) P_{1}(\widetilde{\nabla} \psi)$ is the symmetric product we define in the Appendix of $P_{1}(\widetilde{\nabla} \psi)$ by itself.

Proof From Sect. 3.2 we know that $S$ is a $\operatorname{Spin}(4)$ valued section whose action on $I$ for each $q \in D$, namely $S(q)^{\dagger} I(q) S(q)$, is a rotation (see Sect. 2.3). We explicit this rotation.

The 4-dimensional vector subspace of $\mathcal{C} \mathcal{T}(q)$ isomorphic to $\mathbb{R}_{4,0}^{1}$ by trivialization can be decomposed into two orthogonal planes:

i. The plane generated by the luminance and the temperature components, represented by the bivector $e_{1}(q) e_{4}(q)+e_{2}(q) e_{4}(q)+e_{3}(q) e_{4}(q)$;

ii. The chrominance plane represented by the bivector $e_{1}(q) e_{2}(q)-e_{1}(q) e_{3}(q)+e_{2}(q) e_{3}(q)$.

From this we deduce that the rotation lets the luminance and temperature parts of $I(q)$ invariant and acts on the chrominance plane as a rotation of angle $-h(q)$. That is, it sends the chrominance vector $v(q)$ on the vector $\|v(q)\| \rho(q)$.

Since $P_{1}$ and $P_{2}$ are linear maps, we have

$$
d\left(P_{i}(\psi)\right)=P_{i} d(\psi) \quad i=1,2
$$

From the definition of $\widetilde{\nabla}$, it leads to

$$
P_{i}(\widetilde{\nabla} \psi)=\widetilde{\nabla} P_{i}(\psi)
$$

Then

$$
\begin{aligned}
P_{1}(\widetilde{\nabla} \psi) & =\widetilde{\nabla} P_{1}(\psi) \\
& =\widetilde{\nabla}\left(l\left(e_{1}+e_{2}+e_{3}\right)+t e_{4}\right) \\
& =d l \otimes\left(e_{1}+e_{2}+e_{3}\right)+d t \otimes e_{4}
\end{aligned}
$$


hence

$$
\begin{gathered}
P_{1}(\widetilde{\nabla} \psi) P_{1}(\widetilde{\nabla} \psi)=d l \otimes d l \otimes\left(e_{1}+e_{2}+e_{3}\right)^{2} \\
+\frac{1}{2}(d l \otimes d t+d t \otimes d l) \otimes\left(e_{1}+e_{2}+e_{3}\right) e_{4} \\
+\frac{1}{2}(d t \otimes d l+d l \otimes d t) \otimes e_{4}\left(e_{1}+e_{2}+e_{3}\right) \\
+d t \otimes d t \otimes\left(e_{4}\right)^{2}
\end{gathered}
$$

So, we have

$$
P_{1}(\widetilde{\nabla} \psi) P_{1}(\widetilde{\nabla} \psi)=d l \otimes d l \otimes \lambda+d t \otimes d t \otimes \eta
$$

Simple computations show that $\rho=\sigma / \sqrt{\lambda}$ with

$$
\sigma=\sqrt{2} e_{1}-\frac{\sqrt{2}}{2} e_{2}-\frac{\sqrt{2}}{2} e_{3}
$$

then

$$
\begin{aligned}
P_{2}(\widetilde{\nabla} \psi) & =\widetilde{\nabla}\left(\frac{\|v\| \sigma}{\sqrt{\lambda}}\right) \\
& =\left(d \frac{\|v\|}{\sqrt{\lambda}}\right) \otimes \sigma+\frac{\|v\|}{\sqrt{\lambda}} \widetilde{\nabla} \sigma \\
& =\left(d \frac{\|v\|}{\sqrt{\lambda}}\right) \otimes \sigma
\end{aligned}
$$

Recall that $\|v\|=\frac{\sqrt{2}}{3} \sqrt{\lambda} s$ to obtain

$$
P_{2}(\widetilde{\nabla} \psi)=\frac{\sqrt{2}}{3} d s \otimes \sigma
$$

which leads to

$$
\begin{aligned}
P_{2}(\widetilde{\nabla} \psi) \underline{\otimes} P_{2}(\widetilde{\nabla} \psi) & =\frac{2}{9} d s \otimes d s \otimes \sigma^{2} \\
& =\frac{2}{9} d s \otimes d s \otimes \lambda
\end{aligned}
$$

The section $\gamma$ can be decomposed as

$$
\gamma=\frac{v}{\|v\|} \cdot \rho+\frac{v}{\|v\|} \wedge \rho
$$

Since

$$
\frac{v}{\|v\|} \cdot \rho=\cos (h)
$$

and

$$
\frac{v}{\|v\|} \wedge \rho=\sin (h)\left(\frac{e_{1} e_{2}-e_{1} e_{3}+e_{2} e_{3}}{\left\|e_{1} e_{2}-e_{1} e_{3}+e_{2} e_{3}\right\|}\right)
$$

we have then

$$
\gamma=\cos (h)+\sin (h)\left(\frac{e_{1} e_{2}-e_{1} e_{3}+e_{2} e_{3}}{\left\|e_{1} e_{2}-e_{1} e_{3}+e_{2} e_{3}\right\|}\right)
$$

The expression of $\widetilde{\nabla} \gamma$ is therefore

$$
\begin{gathered}
\widetilde{\nabla} \gamma=-d h \otimes \sin (h)+\cos (h) \widetilde{\nabla} 1 \\
+d h \otimes \cos (h)\left(\frac{e_{1} e_{2}-e_{1} e_{3}+e_{2} e_{3}}{\left\|e_{1} e_{2}-e_{1} e_{3}+e_{2} e_{3}\right\|}\right) \\
+\sqrt{3} \sin (h) \widetilde{\nabla}\left(\frac{e_{1} e_{2}}{\lambda}-\frac{e_{1} e_{3}}{\lambda}+\frac{e_{2} e_{3}}{\lambda}\right)
\end{gathered}
$$

However by definition of $\widetilde{\nabla}$

$$
\widetilde{\nabla} 1=\widetilde{\nabla}\left(\frac{e_{1} e_{2}}{\lambda}\right)=\widetilde{\nabla}\left(\frac{e_{1} e_{3}}{\lambda}\right)=\widetilde{\nabla}\left(\frac{e_{2} e_{3}}{\lambda}\right)=0
$$

and so

$\widetilde{\nabla} \gamma=-d h \otimes \sin (h)+d h \otimes \cos (h)\left(\frac{e_{1} e_{2}-e_{1} e_{3}+e_{2} e_{3}}{\left\|e_{1} e_{2}-e_{1} e_{3}+e_{2} e_{3}\right\|}\right)$

This implies that

$$
\gamma^{\dagger} \widetilde{\nabla} \gamma=\left[\cos (h)-\sin (h)\left(\frac{e_{1} e_{2}-e_{1} e_{3}+e_{2} e_{3}}{\left\|e_{1} e_{2}-e_{1} e_{3}+e_{2} e_{3}\right\|}\right)\right] \times
$$

$\left[\left(-d h \otimes \sin (h)+d h \otimes \cos (h)\left(\frac{e_{1} e_{2}-e_{1} e_{3}+e_{2} e_{3}}{\left\|e_{1} e_{2}-e_{1} e_{3}+e_{2} e_{3}\right\|}\right)\right]\right.$

and

$$
\gamma^{\dagger} \widetilde{\nabla} \gamma=d h \otimes\left(\frac{e_{1} e_{2}-e_{1} e_{3}+e_{2} e_{3}}{\left\|e_{1} e_{2}-e_{1} e_{3}+e_{2} e_{3}\right\|}\right)
$$

Consequently

$$
\left(\gamma^{\dagger} \widetilde{\nabla} \gamma\right) \underline{\otimes}\left(\gamma^{\dagger} \widetilde{\nabla} \gamma\right)=-d h \otimes d h \otimes 1
$$

Let $Z_{1}=\left(Z_{11}, Z_{12}\right)$ and $Z_{2}=\left(Z_{21}, Z_{22}\right)$ be two vector fields on $D$, then by definition of $\chi$ :

$$
\begin{aligned}
\chi\left(Z_{1} \otimes Z_{2}\right) & =d x \underline{\otimes} d x\left(Z_{1} \otimes Z_{2}\right)+d y \underline{\otimes} d y\left(Z_{1} \otimes Z_{2}\right) \\
& +P_{1}(\widetilde{\nabla} \psi) P_{1}(\widetilde{\nabla} \psi)\left(Z_{1} \otimes Z_{2}\right) \\
+ & \frac{9}{2} P_{2}(\widetilde{\nabla} \psi) \underline{\otimes} P_{2}(\widetilde{\nabla} \psi)\left(Z_{1} \otimes Z_{2}\right) \\
& -\kappa \xi\left(\gamma^{\dagger} \widetilde{\nabla} \gamma\right) \underline{\otimes}\left(\gamma^{\dagger} \widetilde{\nabla} \gamma\right)\left(Z_{1} \otimes Z_{2}\right)
\end{aligned}
$$

From what we have shown above, we have

$$
\begin{gathered}
\chi\left(Z_{1} \otimes Z_{2}\right)=d x \underline{\otimes} d x\left(Z_{1} \otimes Z_{2}\right)+d y \underline{\otimes} d y\left(Z_{1} \otimes Z_{2}\right) \\
\quad+d l \otimes d l \otimes \lambda\left(Z_{1} \otimes Z_{2}\right)+d t \otimes d t \otimes \eta\left(Z_{1} \otimes Z_{2}\right) \\
+d s \otimes d s \otimes \lambda\left(Z_{1} \otimes Z_{2}\right)+\kappa \xi d h \otimes d h \otimes 1\left(Z_{1} \otimes Z_{2}\right)
\end{gathered}
$$

Hence,

$$
\begin{gathered}
\chi\left(Z_{1} \otimes Z_{2}\right)=Z_{11} Z_{21}+Z_{12} Z_{22}+d l\left(Z_{1}\right) d l\left(Z_{2}\right) \lambda \\
+d t\left(Z_{1}\right) d t\left(Z_{2}\right) \eta+d s\left(Z_{1}\right) d s\left(Z_{2}\right) \lambda+\kappa \xi d h\left(Z_{1}\right) d h\left(Z_{2}\right)
\end{gathered}
$$

Taking $Z_{1}=Z_{2}=X$, we get the expression of $E$. Similarly, taking $Z_{1}=Z_{2}=Y$, we get the expression of $G$, and from $Z_{1}=X, Z_{2}=Y$ or $Z_{1}=Y, Z_{2}=X$, we get the expression of $F$. 


\subsection{Discretization and parallel transport}

In practice, if an image is represented as a two-dimensional surface $S$ parametrized by a function $\varphi$ on a domain $D$, only the points of $S$ corresponding to integer coordinates points of $D$ are to be taken into account. This implies that derivatives of $\varphi$ must be discreted. For 1D images (i.e. grey level images) Prewitt, Sobel or Canny-Deriche filters can be used to make the approximation. This also may be applied more generally to $\mathrm{nD}$ images by computing the discrete derivatives of each component of $\varphi$.

However, in the vector bundles setting we consider in this paper, such methods are irrelevant since they necessitate to do operations (additions) between objets (multivectors) which don't belong to the same space (they live in different fibers). To solve this problem, we move these objects using the so-called parallel transport so that they can be considered as living in the same fiber.

Let us remind the definition of parallel transport. Let $(E, \pi, M)$ be a vector bundle endowed with a connection $\nabla, \gamma: J \subset \mathbb{R} \rightarrow M$ a curve in $M$ and $v$ a vector of $\pi^{-1}(\gamma(0))$. The parallel transport of $v$ along $\gamma$ is the solution $\tau_{\gamma}(t, v)$ of the following ordinary differential equation:

$$
\left\{\begin{array}{c}
\nabla_{\dot{\gamma}(t)} \tau_{\gamma}=0 \quad \forall t \in J \\
\tau_{\gamma}(0, v)=v
\end{array}\right.
$$

The parallel transport appears classically when dealing with geodesics on manifolds. More precisely, consider the tangent bundle $(T M, \tilde{\pi}, M)$ of a manifold $M$ (not necessiraly equipped with a metric) endowed with a connection $\nabla$. A geodesic is defined as a curve $\gamma: J \rightarrow$ $M$ whose tangent vector field $\dot{\gamma}: J \rightarrow T M$ is parallel along $\gamma$ :

$$
\nabla_{\dot{\gamma}(t)} \dot{\gamma}=0 \quad \forall t \in J
$$

The usual notion of geodesic over surfaces arises from the Levi-Cevita connection[11] induced by the first fundamental form of the surface.

Let us now explain how to compute the coefficients $E$, $F$, and $G$ of Sect. 3.3 when dealing with the integer coordinates points of $D$. In what follows, we use the matricial coordinates system.

We denote

$$
\gamma_{(1,0)}^{(i, j)}, \quad \gamma_{(0,1)}^{(i, j)}, \quad \gamma_{(1,1)}^{(i, j)}, \quad \gamma_{(1,-1)}^{(i, j)}
$$

the classes of curves on $D$ from the point $(i, j)$ that satisfy:

$$
\dot{\gamma}_{(1,0)}^{(i, j)}(t)=(1,0), \quad \dot{\gamma}_{(1,0)}^{(i, j)}(t)=(0,1),
$$

$$
\dot{\gamma}_{(1,1)}^{(i, j)}(t)=(1,1), \quad \dot{\gamma}_{(1,-1)}^{(i, j)}(t)=(1,-1)
$$

for all $t$. The corresponding parallel transports (with respect to $\widetilde{\nabla}$ )

$$
\tau_{(1,0)}^{(i, j)}(t, .), \quad \tau_{(0,1)}^{(i, j)}(t, .), \quad \tau_{(1,1)}^{(i, j)}(t, .), \quad \tau_{(1,-1)}^{(i, j)}(t, .)
$$

are linear maps from $\pi^{-1}(i, j)$ to $\pi^{-1}\left(\gamma_{(1,0)}^{(i, j)}(t)\right)$ resp. $\pi^{-1}\left(\gamma_{(0,1)}^{(i, j)}(t)\right), \pi^{-1}\left(\gamma_{(1,1)}^{(i, j)}(t)\right)$ and $\pi^{-1}\left(\gamma_{(1,-1)}^{(i, j)}(t)\right)$.

By definition of $\widetilde{\nabla}$, if $\gamma$ is one of the preceding classes of curves and $w$ is a vector of $\pi^{-1}(\gamma(0))$, i.e

$$
\begin{gathered}
w=w_{1} e_{1}(\gamma(0))+w_{2} e_{2}(\gamma(0)) \\
+w_{3} e_{3}(\gamma(0))+w_{4} e_{4}(\gamma(0))
\end{gathered}
$$

then the parallel transport of $w$ at $\pi^{-1}(\gamma(t))$ is the vector

$$
\begin{gathered}
w_{1} e_{1}(\gamma(t))+w_{2} e_{2}(\gamma(t)) \\
+w_{3} e_{3}(\gamma(t))+w_{4} e_{4}(\gamma(t))
\end{gathered}
$$

Let us consider the vector

$$
\begin{aligned}
\tau_{1}(i, j) & =\frac{1}{8}\left\{\tau_{(1,-1)}^{(i+1, j-1)}(-1, \psi)+2 \tau_{(1,0)}^{(i+1, j)}(-1, \psi)\right. \\
& +\tau_{(1,1)}^{(i+1, j+1)}(-1, \psi)-\tau_{(1,1)}^{(i-1, j-1)}(1, \psi) \\
& \left.-2 \tau_{(1,0)}^{(i-1, j)}(1, \psi)-\tau_{(1,-1)}^{(i-1, j+1)}(1, \psi)\right\}
\end{aligned}
$$

and the scalar

$\tau_{2}(i, j)=\frac{1}{8}\{\arccos (a \cdot b)+2 \arccos (c \cdot d)+\arccos (e \cdot f)\}$

where

$$
\begin{gathered}
a=\frac{\tau_{(1,1)}^{(i-1, j-1)}(1, v)}{\left\|\tau_{(1,1)}^{(i-1, j-1)}(1, v)\right\|} \quad b=\frac{\tau_{(1,-1)}^{(i+1, j-1)}(-1, v)}{\left\|\tau_{(1,-1)}^{(i+1, j-1)}(-1, v)\right\|} \\
c=\frac{\tau_{(1,0)}^{(i-1, j)}(1, v)}{\left\|\tau_{(1,0)}^{(i-1, j)}(1, v)\right\|} \quad d=\frac{\tau_{(1,0)}^{(i+1, j)}(-1, v)}{\left\|\tau_{(1,0)}^{(i+1, j)}(-1, v)\right\|} \\
e=\frac{\tau_{(1,-1)}^{(i-1, j+1)}(1, v)}{\left\|\tau_{(1,-1)}^{(i-1, j+1)}(1, v)\right\|} \quad f=\frac{\tau_{(1,1)}^{(i+1, j+1)}(-1, v)}{\left\|\tau_{(1,1)}^{(i+1, j+1)}(-1, v)\right\|}
\end{gathered}
$$

These are elements of $\pi^{-1}(i, j)$.

The vector $\tau_{1}(i, j)$ (resp. the scalar $\tau_{2}(i, j)$ ) is a discrete approximation of $\widetilde{\nabla} \psi$ (resp. $d h \otimes 1$ ) in the direction given by the tangent vector of coordinates $(1,0)$ at $(i, j)$. We obtain thus a discrete version of the coefficient $G$ of the fundamental form I, namely

$$
G_{d}=1+\left(P_{1}\left(\tau_{1}\right)\right)^{2}+\frac{9}{2}\left(P_{2}\left(\tau_{1}\right)\right)^{2}+\kappa \xi \tau_{2}^{2}
$$


Detailing the computation of $G_{d}$, Clifford algebras reveal all their assets (at least, the three mentionned in the Introduction). First, we exploit the efficiency of the geometric calculus to compute $\tau_{1}$ and $\tau_{2}$ in a concise way. Indeed, we don't need matrix representation to compute the rotations $S^{\dagger} I S$ but only algebraic operations using spinors. The computation is trivial when remark that, as

$$
\left(\frac{e_{1} e_{2}-e_{1} e_{3}+e_{2} e_{3}}{\left\|e_{1} e_{2}-e_{1} e_{3}+e_{2} e_{3}\right\|}\right)^{2}=-1,
$$

we have

$$
S=\cos \left(\frac{h}{2}\right)-\sin \left(\frac{h}{2}\right) \frac{e_{1} e_{2}-e_{1} e_{3}+e_{2} e_{3}}{\left\|e_{1} e_{2}-e_{1} e_{3}+e_{2} e_{3}\right\|}
$$

From a generalized directional Sobel filter applied to $S^{\dagger} I S$ using parallel transport, we obtain $\tau_{1}$ that is a discrete approximation of the vector-valued section

$$
l_{y}\left(e_{1}+e_{2}+e_{3}\right)+t_{y} e_{4}+\frac{\sqrt{2}}{3} s_{y} \sigma
$$

Let us remark that the angle between two chrominance vectors represents the hue difference between the two corresponding colors. This allows us to compute hue discrete derivatives efficiently. Let $(i, j) \in D$, we first compute chrominance vectors of the $3 \times 3$ neighborhood of $(i, j)$ using the rejection operator of geometric calculus (Sect. 2.1), and we map them into the fiber $\pi^{-1}(i, j)$ by parallel transport. Then, computing some angles from the inner product of the normalized vectors, we derive a kind of Sobel mask and we obtain $\tau_{2}(i, j)$, that is a dicrete approximation of $h_{y}$ at $(i, j)$. The way we get this approximation avoids to compute discrete derivatives of the function (that is the problem of measuring distances on $S^{1}$ ).

As a second asset, we see that all the information we need to compute $G_{d}$ may be included into a single multivector-valued section

$$
\tau:=\tau_{1}+\tau_{2}
$$

which contains a scalar and a vector part. Inversely, from the multivector $\tau$, we get

$$
\tau_{1}=\frac{t(\tau)-\alpha(\tau)}{2}
$$

and

$$
\tau_{2}=\frac{t(\tau)+\alpha(\tau)}{2}
$$

where $t$ and $\alpha$ are the extensions of the two morphisms defined in Sect. 2.1 to $\mathcal{C} \mathcal{T}(D)$.
At last, we use the computability of the orthogonal projection operator (Sect. 2.1), and the property of Clifford algebras that a squared vector gives its squared norm in the associated quadratic vector space. Indeed we may compute directly $G_{d}$ from $\tau$ and the functions $\xi$ and $\kappa$, where $\xi$ is fixed (Sect. 2.3) and $\kappa$ has to be determined (depends on the chosen edge detection) by the following formula. We have

$$
G_{d}=1+P_{1}\left(\frac{t(\tau)-\alpha(\tau)}{2}\right)^{2}+\frac{9}{2} P_{2}\left(\frac{t(\tau)-\alpha(\tau)}{2}\right)^{2}
$$

$$
+\kappa \xi\left(\frac{t(\tau)+\alpha(\tau)}{2}\right)^{2}
$$

In other words, we compute a discrete approximation of

$$
1+\lambda l_{y}^{2}+\lambda s_{y}^{2}+\kappa \xi h_{y}^{2}+\eta t_{y}^{2}
$$

In the same way, we get a discrete version of the coefficients $E$ and $F$.

\section{Applications}

We propose three applications of the computation of $\chi$ of Sect. 3.3: First we compare our approach with the method developed by Di Zenzo, then we focus on detecting edges in color images with respect to a given hue interval and saturation levels of colors. The third application is devoted to detect edges in color-infrared images with constraints on color and temperature. Finally we describe briefly further work on color edges analysis, related to the classification of edges in function of they are due to shadows, highlights, or transitions between objects.

\subsection{Comparison with the Di Zenzo gradient}

In this part, we first show that we obtain the metric of the Di Zenzo's approach by derivating the section representing a color image with the connection $\widetilde{\nabla}$ and taking a well-chosen metric on the fiber bundle. Then, we explicit the difference between the metric of Di Zenzo and the one given by Proposition 2 .

Di Zenzo's approach of edge detection is to consider a color image as a 2-dimensional surface parametrized by

$$
\varphi:(x, y) \longmapsto(x, y, r(x, y), g(x, y), b(x, y))
$$


embedded in $\left(\mathbb{R}^{5},\|\|_{2}\right)$. Coefficients of the first fundamental form are therefore given by:

$$
\begin{gathered}
E_{D Z}=1+\left(r_{x}\right)^{2}+\left(g_{x}\right)^{2}+\left(b_{x}\right)^{2} \\
F_{D Z}=r_{x} r_{y}+g_{x} g_{y}+b_{x} b_{y} \\
G_{D Z}=1+\left(r_{y}\right)^{2}+\left(g_{y}\right)^{2}+\left(b_{y}\right)^{2}
\end{gathered}
$$

Let us consider the Clifford bundle $(\mathcal{C T}(D), \pi, D)$ constructed from the metric

$$
\left(\begin{array}{cccc}
1 / 3 & 0 & 0 & 0 \\
0 & 1 / 3 & 0 & 0 \\
0 & 0 & 1 / 3 & 0 \\
0 & 0 & 0 & \eta
\end{array}\right)
$$

where $\eta$ is any strictly positive function, so that the fiber bundle is well-defined.

Remark 1 As we deal with color images, the values of $\eta$ will not affect the result of the edge detection. Note that we could have constructed a Clifford bundle from a vector bundle of rank 3 . In this paper, we have chosen to consider a color image as the color part of a color/infrared image so that to make our method relevant to deal more generally with $\mathrm{nD}$ images edge detection.

Let $I(q)=r(q) e_{1}(q)+g(q) e_{2}(q)+b(q) e_{3}(q)+t(q) e_{4}(q)$ be a color/infrared image, seen as a section of $\mathcal{C T}(D)$, and let us denote $I_{\text {col }}(q)=r(q) e_{1}(q)+g(q) e_{2}(q)+b(q) e_{3}(q)$ its color part, that is

$$
I_{c o l}=I \cdot\left(e_{1} e_{2} e_{3}\right)\left(e_{1} e_{2} e_{3}\right)^{-1}
$$

Then, we have

$$
\widetilde{\nabla} I_{c o l}=d r \otimes e_{1}+d g \otimes e_{2}+d b \otimes e_{3}
$$

and the symmetric product of $\widetilde{\nabla}$ by itself (see Appendix) is therefore:

$\widetilde{\nabla} I_{c o l} \widetilde{\nabla} I_{c o l}=d r \otimes d r \otimes \frac{1}{3}+d g \otimes d g \otimes \frac{1}{3}+d b \otimes d b \otimes \frac{1}{3}$

So, using the same notations as in Proposition 2, and considering the symmetric tensor of rank $2 \chi_{D Z}$ defined by

$$
\chi_{D Z}=d x \underline{\otimes} d x+d y \underline{\otimes} d y+3 \widetilde{\nabla} I_{c o l} \tilde{\nabla} I_{c o l}
$$

we get

$$
\begin{gathered}
E_{D Z}=\chi_{D Z}(X \otimes X) \quad F_{D Z}=\chi_{D Z}(X \otimes Y) \\
G_{D Z}=\chi_{D Z}(Y \otimes Y)
\end{gathered}
$$

Furthermore we can split $I_{c o l}$ into

$$
\left(I_{\text {col }} \cdot \mu\right) \mu^{-1}+\left(I_{\text {col }} \wedge \mu\right) \mu^{-1}=l\left(e_{1}+e_{2}+e_{3}\right)+v
$$

Since

$$
\widetilde{\nabla} I_{c o l}=d l \otimes\left(e_{1}+e_{2}+e_{3}\right)+\widetilde{\nabla} v
$$

we see that the method of Di Zenzo deals with the derivative $\widetilde{\nabla} v$ of the chrominance vector which, by definition of $\widetilde{\nabla}$, corresponds to the usual derivative of the vector-valued function $v$. Moreover, since $\left(e_{1}+e_{2}+e_{3}\right)$ and $v$ are orthogonal, then

$$
\widetilde{\nabla} I_{c o l} \widetilde{\nabla} I_{c o l}=d l \otimes d l \otimes 1+\widetilde{\nabla} v \widetilde{\nabla} v
$$

and

$$
\chi_{D Z}=d x \underline{\otimes} d x+d y \underline{\otimes} d y+d l \otimes d l \otimes 3+3 \widetilde{\nabla} v \widetilde{\nabla} v
$$

From simple computations, we have

$$
\begin{aligned}
& E_{D Z}=1+3\left(l_{x}\right)^{2}+v_{x}^{2} \\
& F_{D Z}=3\left(l_{x} l_{y}\right)+v_{x} \cdot v_{y} \\
& G_{D Z}=1+3\left(l_{y}\right)^{2}+v_{y}^{2}
\end{aligned}
$$

If we apply now Proposition 2 for $\psi=S^{\dagger} I_{\text {col }} S$ in the context of this fiber bundle, the corresponding coefficients are given by

$$
\begin{gathered}
E=1+\left(l_{x}\right)^{2}+\left(s_{x}\right)^{2}+\kappa \xi\left(h_{x}\right)^{2} \\
F=l_{x} l_{y}+s_{x} s_{y}+\kappa \xi h_{x} h_{y} \\
G=1+\left(l_{y}\right)^{2}+\left(s_{y}\right)^{2}+\kappa \xi\left(h_{y}\right)^{2}
\end{gathered}
$$

and variations of the chrominance part are given by both variations of saturation and hue components.

We conclude that these two methods differ first by the weight of the luminance part, then by the metric of the chrominance part, which is Euclidean for the Di Zenzo's method, and Riemannian for the method we propose (the coefficient $\xi$ of the metric associated to the hue component varies with the saturation).

Let us consider as an example the case where the hue is locally constant, i.e. $d h=0$, then

$$
\widetilde{\nabla} v=d(\|v\|) \otimes \frac{v}{\|v\|}
$$

and

$$
\widetilde{\nabla} v \widetilde{\nabla} v=d(\|v\|) \otimes d(\|v\|) \otimes 1=d s \otimes d s \otimes \frac{2}{9}
$$

The coefficients for the Di Zenzo gradient are therefore

$$
\begin{gathered}
E_{D Z}=1+3\left(l_{x}\right)^{2}+\frac{2}{3} s_{x}^{2} \\
F_{D Z}=3\left(l_{x} l_{y}\right)+\frac{2}{3} s_{x} s_{y}
\end{gathered}
$$




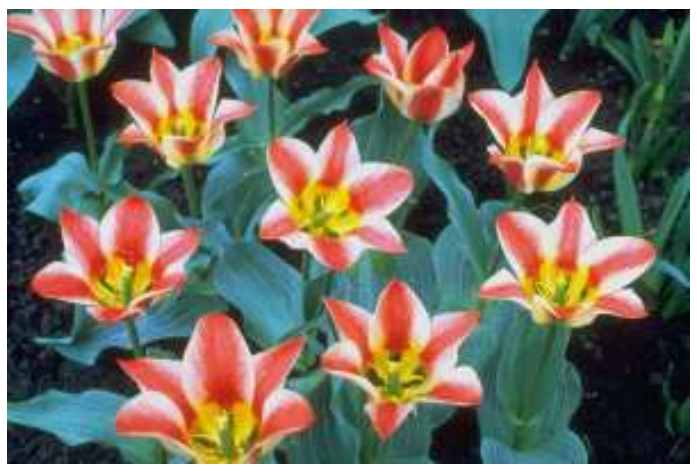

Fig. 1 Original image

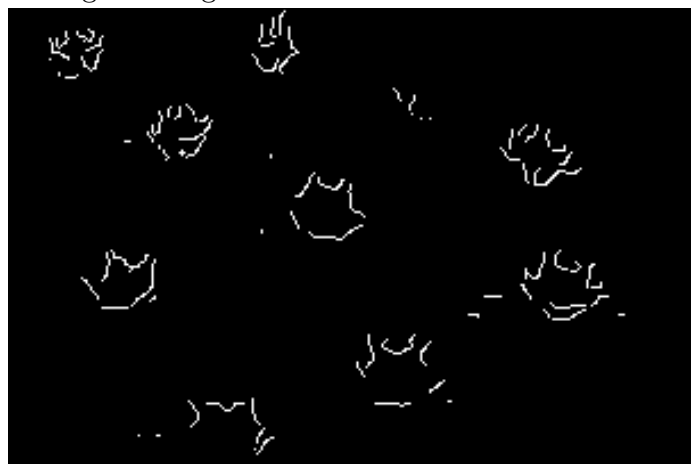

Fig. 2 Edge detection in the hue interval Red-Yellow

$$
G_{D Z}=1+3\left(l_{y}\right)^{2}+\frac{2}{3}\left(s_{y}\right)^{2}
$$

while those we have defined are

$$
\begin{gathered}
E=1+\left(l_{x}\right)^{2}+\left(s_{x}\right)^{2} \\
F=l_{x} l_{y}+s_{x} s_{y} \\
G=1+\left(l_{y}\right)^{2}+\left(s_{y}\right)^{2}
\end{gathered}
$$

We can see in this very particular case that the two methods differ from the ratio

weight of luminance/weight of saturation

in the measure of the variations of a color image.

However, let us mention the problem of determining which one of the two metrics is the most perceptual. A solution to solve this problem could be to compare these two metrics with the perceptual metric of $L a b$ pull-backed on $R G B$.

4.2 Color edge detection with respect to hue intervals and saturation levels

The aim of this application is to detect edges characterizing transitions between highly saturated colors of a given hue interval.
Let $I_{c o l}(q)=r(q) e_{1}(q)+g(q) e_{2}(q)+b(q) e_{3}(q)$ be a section of $\mathcal{C} \mathcal{T}(D)$ corresponding to the color part of a color/infrared image, where the coefficients of the metric generating $\mathcal{C} \mathcal{T}(D)$ are defined as follows. As for this application, we don't deal with temperature variations, $\eta$ can be taken as any strictly positive function again. Moreover, since we only consider hue variations, the choice of $\kappa$ influences the result, and $\lambda$ doesn't. Therefore let us take $\lambda$ be any strictly positive function, high enough so that the norm of a vector at each fiber is numerically computable. Indeed, we only have to compute the part which contains the information about hue variations. It is given by $1+\kappa \xi \tau_{2}^{2}$ (see Sect. 3.4). By definition of $\tau_{2}$, we compute norms of vectors, which depend on the value of $\lambda$.

In the continuous setting, this information is given by

$$
d x \underline{\otimes} d x+d y \underline{\otimes} d y-\kappa \xi\left(\gamma^{\dagger} \widetilde{\nabla} \gamma\right) \underline{\otimes}\left(\gamma^{\dagger} \widetilde{\nabla} \gamma\right)
$$

This latter can be viewed as the first fundamental form of the surface parametrized by

$$
\varphi:(x, y) \mapsto(x, y, h)
$$

embedded in $\mathbb{R}^{3}$ equipped with the metric

$$
\left(\begin{array}{ccc}
1 & 0 & 0 \\
0 & 1 & 0 \\
0 & 0 & \kappa \xi
\end{array}\right)
$$

Let us now detail the construction of the function $\kappa$.

In the sequel, for $q \in D$, we consider the domain

$$
\Omega(q)=\left\{(x, y),\|(x, y)-q\|_{\infty} \leq 1\right\}
$$

Let $\theta$ in $[0,2 \pi]$. The unit chrominance vector $v_{\theta}$ of hue $\theta$ is

$$
v_{\theta}=\cos (\theta) \rho+\sin (\theta) \rho B
$$

where $B$ is the unit bivector coding the chrominance plane. The angular distance $\delta$ between two hues $h_{3}$ and $h_{4}$ may be computed using the corresponding unit chrominance vectors

$$
\delta\left(h_{3}, h_{4}\right)=\arccos \left(v_{h_{3}} \cdot v_{h_{4}}\right)
$$

Let us note $h_{1}$ and $h_{2}$ the two hues representing boundaries of the given hue interval. We set $\kappa(q)=1$ if

$$
\max _{x \in \Omega(q)}\left(\max \left(\delta\left(h(x), h_{1}\right), \delta\left(h(x), h_{2}\right)\right)\right) \leq \delta\left(h_{1}, h_{2}\right)
$$

and extend $\kappa$ into a derivable function on $[0, \pi]$ that is strictly decreasing on $\left[\delta\left(h_{1}, h_{2}\right), \pi\right]$. 
In the illustration of this application, $h_{1}=0$ is the red hue, $h_{2}=\pi / 3$ is the yellow hue, and $\kappa$ equals 0 for any value greater than $\pi / 3$.

As we can see Fig. 2, we principally detect edges of Fig. 1 inside petals. Let us explain this result. First, due to the definition of $\kappa$, as soon as there is a pixel in the neighborhood of a point $q$ in $D$ whose hue is not in the interval red-yellow, color variations at $q$ are not detected. That's why edges involving green color, as on stems, are not detected. Then, one may find inside petals variations between yellow and red hues, which implies that $\kappa d h$ is maximal. Moreover, these colors have almost a full saturation, so $\xi$ almost equals 1 . In the same way, variations where one of the colors has low saturation are not detected, since $\xi$ decreases strongly when the surrounding saturation declines. This explains why transitions between colors of yellow or red hue and colors which look white are not detected.

\subsection{Color/infrared images edge detection}

We present here an edge detection combining information given by both color and temperature variations of a scene. It seems to be particulary suitable to detect humans. Indeed, humans may be characterized by their temperature and the color of their skin. Then, variations of temperature provide transitions between humans and their environment. Conjointly, color variations allow us to have a better description of humans, by detecting edges inside the regions they determine.

Let us consider the following situation: a man is standing in front of a wall and is handing a cup of hot coffee (see Fig. 3). We want to detect edges corresponding to regions of temperature similar to the temperature of the human body and of color similar to the color of the skin, that is his face and his arm (see Fig. 3).

First, let us consider this color-infrared image as a function $D \longrightarrow \mathbb{R}_{4,0}$

$$
I(q)=r(q) e_{1}+g(q) e_{2}+b(q) e_{3}+t(q) e_{4}
$$

Let $\zeta=\zeta_{1} e_{1}+\zeta_{2} e_{2}+\zeta_{3} e_{3}=I_{c o l}(x, y)$ for $(x, y)$ located on the face or on the arm and chosen arbitrarily. Therefore $\zeta$ is a relevant representant of the color of the skin.

Then, we proceed as follows:

We compute the function $\zeta I$, which may be decomposed as the sum of a scalar and a bivector function:

$$
\zeta I=\zeta \cdot I+\zeta \wedge I
$$

From the information given by $\zeta \cdot I$ and $\zeta \wedge I$, we construct the metric generating a Clifford bundle $\mathcal{C} \mathcal{T}(D)$, and the function $\kappa$. Then, we consider the color/infrared image as a section of this fiber bundle

$$
I(q)=r(q) e_{1}(q)+g(q) e_{2}(q)+b(q) e_{3}(q)+t(q) e_{4}(q)
$$

and compute the tensor $\chi$ in this setting. We get then the coefficients of the first fundamental form of the surface parametrized by

$$
\varphi:(x, y) \longmapsto(x, y, l(x, y), s(x, y), h(x, y), t(x, y))
$$

embedded in $\mathbb{R}^{6}$ equipped with metric

$$
\left(\begin{array}{ll}
1 & 0 \\
0 & 1
\end{array}\right) \oplus\left(\begin{array}{cccc}
\lambda(p) & 0 & 0 & 0 \\
0 & \lambda(p) & 0 & 0 \\
0 & 0 & \kappa(p) \xi(p) & 0 \\
0 & 0 & 0 & \eta(p)
\end{array}\right)
$$

Let us detail how we proceed to construct the metric generating $\mathcal{C} \mathcal{T}(\mathcal{D})$.

If we denote by $\alpha$ the function which gives for each $q \in$ $D$ the angle between $\zeta$ and $I_{\text {col }}(q)$, we have $|\tan (\alpha)|=$

$$
\frac{\|\zeta\|\left\|I_{c o l}\right\||\sin (\alpha)|}{\|\zeta\|\left\|I_{c o l}\right\||\cos (\alpha)|}=\frac{\left\|<\zeta I>_{2} \cdot\left(e_{1} e_{2} e_{3}\right)\left(e_{1} e_{2} e_{3}\right)^{-1}\right\|}{\left|<\zeta I>_{0}\right|}
$$

From $|\tan (\alpha)|$, we get $|\alpha|$ since $-\pi / 2 \leq \alpha \leq \pi / 2(\zeta$ and $I_{\text {col }}(q)$ are vectors in the $R G B$ cube).

Note that $|\alpha|$ appears to be a suitable measure of similarity to skin color. Indeed, we can see on Fig. 3 that the skin color is darker on the parts corresponding to the beard and shadow on the arm; luminance and saturation are decreased, hue is almost unchanged. Moreover, in $R G B$ space, decrease or increase of luminance and saturation with the same scale parameter combined with unchanged hue is represented by an homothety of the corresponding color vector. More precisely, simple computations show that

$$
l(k a)=k l(a) \quad s(k a)=k s(a) \quad h(k a)=h(a)
$$

for $k \in \mathbb{R}$ and $a \in R G B$ such that $k a \in R G B$.

To summarize, we say that color variations located on the face and the arm may be, roughly speaking, assimilated to homotheties. Then, the choice of $|\alpha|$ to characterize them arises from the invariance of $\alpha$ with respect to homotheties.

Then, we determine thresholds on $|\alpha|$ and $t$ respectively $\alpha_{0}$ and $t_{0}$ that define regions of interest using the following map (see Fig. 3). 

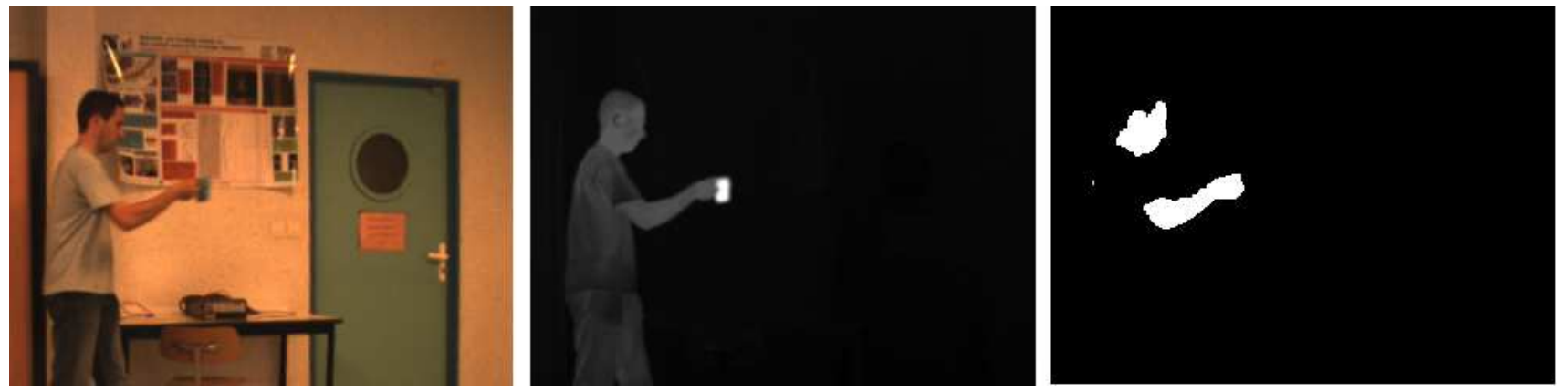

Fig. 3 left and center: color and temperature information of the scene - right: regions where $g_{1}=1$ in white

We set $g_{1}(q)=1$ if

$$
\min _{x \in \Omega(q)}|\alpha(x)| \leq \alpha_{0} \quad \text { and } \max _{x \in \Omega(q)} t(x) \geq t_{0}
$$

and extend $g_{1}$ into a derivable function on $[0, \pi / 2] \times$ $[0,255]$ that is strictly decreasing with respect to the first variable on $\left[\alpha_{0}, \pi / 2\right]$, and with respect to the second variable on $\left[0, t_{0}\right]$. Moreover we ask $g_{1}$ to be strictly positive since the metric $g$ we take to generate $\mathcal{C T}(D)$ is

$$
g:=\left(\begin{array}{cccc}
g_{1} & 0 & 0 & 0 \\
0 & g_{1} & 0 & 0 \\
0 & 0 & g_{1} & 0 \\
0 & 0 & 0 & \nu g_{1}
\end{array}\right)
$$

The role of $\nu$ is to control the weight of temperature variations in the image.

Moreover, we choose $\kappa=255 g_{1} / \pi$ so that the hue component has the same weight in the measure of variations of $I$ as the two other color components, that are luminance and saturation.

Fig. 4 shows the results of edge detections for different values of $\nu$, increasing from the left to the right. On the left, $\nu$ is taken small so that the edge detection is similar to a color edge detection with constraints on color and temperature. We see that transitions between the man and his environment are not well detected on some parts (the region around the nose and the transition between the hand and the cup of coffee). For the two pictures at the center, both color and temperature variations are taken into account. In the case of $\nu=10$, we still detect color details as the eye, the beard and shadow on the arm. Moreover, we see that transitions between the man and his environment are better localized. Increasing again the weight of temperature variations $(\nu=30)$, we don't detect anymore color details, but only edges characterizing the frontiers of the regions of interest (see Fig. 3). The last picture shows an edge detection for $\nu$ taken high, that is an edge detection which can be assimilated to a temperature edge detection with respect to color and temperature constraints. We remark that the frontier between face and hair is not well detected. This comes from the fact that this region represents small temperature variations, as we can see on the temperature information of the scene (Fig. 3).

4.4 One more example of possible applications: color edge analysis

As mentionned in the Introduction, many other applications can be considered from the general setting we have described before. To conclude this section we discuss one of them. Since the approach is slightly different and necessitates more mathematical developments, we only sketch the main steps of the process. Details will appear elsewhere [2].

The problem is the following one. In [15] Gevers \& al. classify edges of a color image according to they are due to the presence of different objects, to shadows, or to highlights, under the assumptions of white illumination and neutral interface reflection. Their method is based on color models they introduced in [16] for the purpose of color objects recognition.

We propose here to show how to obtain similar results (under the same assumptions) using the mathematical formalism of Sect. 3. Unlike other edge detections we have presented in this paper, the metrics we construct don't depend on the values of the image, but of the values of its derivatives.

We proceed in several steps:

First, we consider a nD image as a section of a Clifford bundle $\left(\mathcal{C T}_{1}(D), \pi_{1}, D\right)$ generated by a metric

$$
g=\left(\begin{array}{llll}
g_{1} & & & \\
& g_{2} & & \\
& & \ddots & \\
& & & g_{n}
\end{array}\right)
$$



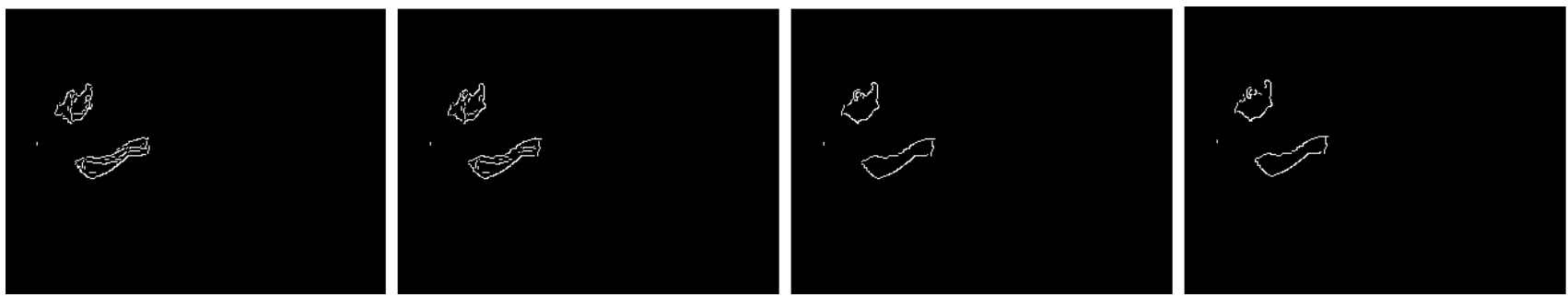

Fig. 4 Color/temperature edge detections for different values of $\nu$. From left to right: $\nu=0.001,10,30,100$

The main difference with the framework developed in Sec. 3 is that a $\mathrm{nD}$ image takes now the following form

$I(q)=I_{1}(q) \frac{e_{1}(q)}{\sqrt{g_{1}(q)}}+I_{2}(q) \frac{e_{2}(q)}{\sqrt{g_{2}(q)}}+\ldots+I_{n}(q) \frac{e_{n}(q)}{\sqrt{g_{n}(q)}}$

In other words, we choose a trivialization that makes the fibers isomorphic (as algebras) to the typical fiber. Therefore we can define a covariant derivative $\widetilde{\nabla}$ which is compatible with the Clifford product, i.e

$$
\widetilde{\nabla}(M N)=\widetilde{\nabla}(M) N+M \widetilde{\nabla}(N) \quad \forall M, N \in \Gamma(\mathcal{C} \mathcal{T}(D))
$$

For the purpose of this application, we deal with $n=3$ for color images.

From $I$, we derive the section $S$ defined by

$$
S=I+\left(\left(\frac{e_{1}}{\sqrt{g_{1}}}+\frac{e_{2}}{\sqrt{g_{2}}}+\frac{e_{3}}{\sqrt{g_{3}}}\right) \wedge I\right) B
$$

where $B$ is a bivector-valued section coding the current hue.

Then, differentiating $S$ with $\widetilde{\nabla}$ leads to a 1 -form with values in the Clifford bundle, i.e $\widetilde{\nabla}(S) \in \Gamma\left(T^{*} D \otimes\right.$ $\left.\mathcal{C} \mathcal{T}_{1}(D)\right)$. It may be written as

$$
\widetilde{\nabla}(S)=\widetilde{\nabla}(S)_{0}+\widetilde{\nabla}(S)_{1}+\widetilde{\nabla}(S)_{2}
$$

where $\widetilde{\nabla}(S)_{j}$ is an element of $\Gamma\left(T^{*} D \otimes \mathcal{C} \mathcal{T}_{1}(D)\right)$ of degree $j$. Each of them codes a particular information. From the scalar and bivector parts, we construct metrics $h_{i}$ generating Clifford bundles $\left(\mathcal{C} \mathcal{T}_{i}(D), \pi_{i}, D\right)$ over D.

At last, by mapping the vector part into each one of the bundles $\left(T^{*} D \otimes \mathcal{C} \mathcal{T}_{i}(D), p_{i}, D\right)$ where $p_{i}$ denotes the projection maps, we can measure the variations of $I$ with respect to the metrics $h_{i}$. In this way we obtain edge detections of Fig. 5.

\section{Conclusion}

In this paper, we have proposed a new framework for $\mathrm{nD}$ image processing, namely Clifford algebras bundles
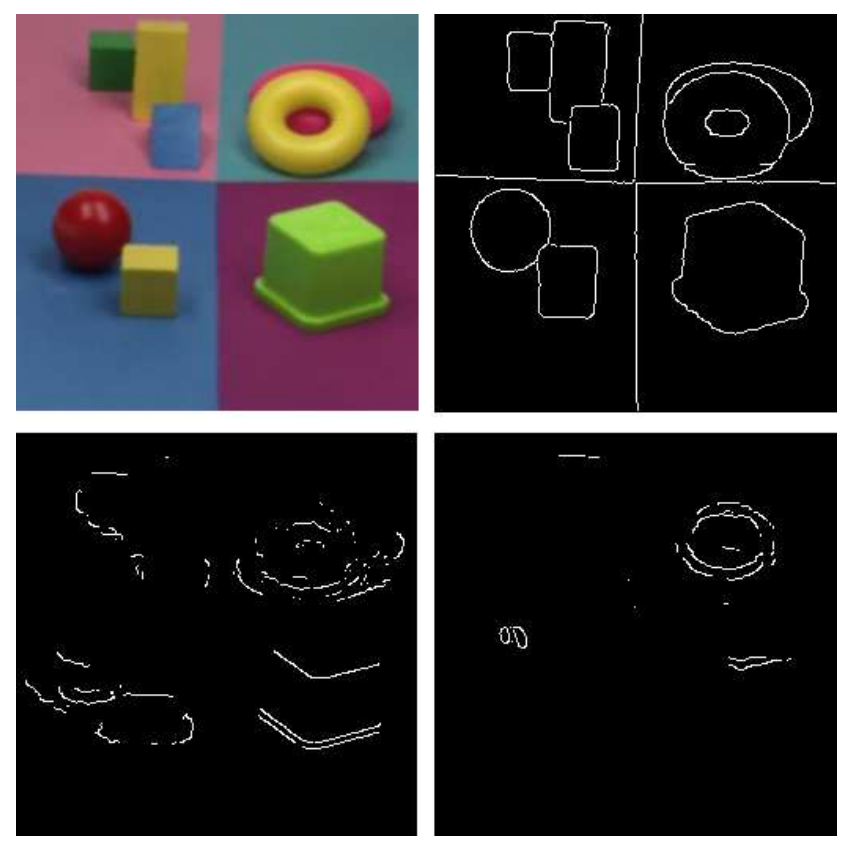

Fig. 5 Clockwise from upper left: original image, objects, highlights, shadows

where the typical fiber is the Clifford algebra associated to the Euclidean space of acquisition. $\mathrm{A} \mathrm{nD}$ image is then considered as a section. The paper deals in particular with the reformulation, in this fiber bundle setting, of a well-known method for $\mathrm{nD}$ images edge detection, based on the computation of the first fundamental form of a surface embedded in a metric space. For this, we have introduced a connection on the fiber bundle, and used corresponding covariant derivative and parallel transport on sections derived from the section defined by the image. We have treated the special case of color/infrared images where the space of acquisition is $R G B T$, and the metric space containing the surface is $L S H T$. We have used the richness of the structure of Clifford algebras and the computability of the operations in two ways detailled as follows. First, we have recovered the first fundamental form by computations in $R G B T$, never computing explicitely derivatives of luminance, saturation, hue or temperature, whereas the 
approach based on surfaces does. Moreover, we have obtained some geometric information about the images considered, to determine some particular metrics leading to detect specific edges. It is therefore natural to envisage that this can be done whatever the metric space considered, whatever the dimension of the images. As it can be seen in Sect. 4.4, further work is devoted to develop more connections, sections and metrics to detect other kinds of edges. Note that Clifford algebras provide a convenient framework to deal with several geometries [28]. Therefore, our framework may be extended to Clifford bundles where the typical fiber is associated to a non-Euclidean vector space, in such a way that geometric information from which metrics are constructed should come from non-Euclidean geometric relations. At last, let us mention that fiber bundle theory generalizes the one of surfaces and manifolds, then it may be beneficial to regard it more closely, in view of other applications to image processing.

\section{Appendix}

We state more precisely in this Appendix how are defined the tensor product of $\mathcal{C} T(D)$-valued 1-forms and the symmetric product used in particular in Proposition 2.

Let $(\mathcal{C} T(D), D, \pi)$ be the fiber bundle introduced in Sect. 3.2. We denote $\mathcal{A}$ the ring of $\mathbb{R}$-valued functions defined on $D$ and $\mathcal{B}$ the ring $\Gamma(\mathcal{C} \mathcal{T}(D))$ of sections of $\mathcal{C T}(D)$.

\section{Proposition 3 The couple}

$$
\left(\Gamma_{1}, \varphi\right):=\left(\Gamma\left(T^{*} D \otimes_{\mathcal{A}} T^{*} D \otimes_{\mathcal{A}} \mathcal{C} \mathcal{T}(D)\right), \varphi\right)
$$

is a solution of the universal problem defining the tensor product of the $\mathcal{B}$-bimodule

$$
\Gamma_{0}:=\Gamma\left(T^{*} D \otimes_{\mathcal{A}} \mathcal{C} \mathcal{T}(D)\right)
$$

with itself, where $\varphi$ is the application from $\Gamma_{0} \times \Gamma_{0}$ to $\Gamma_{1}$ defined by

$$
\left(\left(\omega_{1} \otimes m_{1}\right),\left(\omega_{2} \otimes m_{2}\right)\right) \longmapsto\left(\omega_{1} \otimes \omega_{2}\right) \otimes\left(m_{1} m_{2}\right)
$$

Proof Since $\mathcal{B}$ a $\mathcal{A}$-algebra, there exists a $\mathcal{B}$-bimodule structure on $\Gamma_{0}$

$$
\begin{aligned}
& \mathcal{B} \times \Gamma_{0} \longrightarrow \Gamma_{0} \quad \Gamma_{0} \times \mathcal{B} \longrightarrow \Gamma_{0} \\
& (b, X \otimes c) \longmapsto X \otimes b c \quad(X \otimes c, b) \longmapsto X \otimes c b
\end{aligned}
$$

We consider the following universel problem:
We search a couple

$$
\left(\Gamma_{0} \otimes_{\mathcal{B}} \Gamma_{0}, \phi\right)
$$

where $\Gamma_{0} \otimes_{\mathcal{B}} \Gamma_{0}$ is a $\mathcal{B}$-bimodule and

$$
\phi: \Gamma_{0} \times \Gamma_{0} \longrightarrow \Gamma_{0} \otimes_{\mathcal{B}} \Gamma_{0}
$$

is left $\mathcal{B}$-linear in the first variable and right $\mathcal{B}$-linear in the second variable with

$$
\phi(x f, y)=\phi(x, f y)
$$

for all $x$ and $y$ in $\Gamma_{0}$ and $f$ in $\mathcal{B}$ such that:

For each $\mathcal{B}$-bimodule $N$ and each map

$$
\eta: \Gamma_{0} \times \Gamma_{0} \longrightarrow N
$$

which is left $\mathcal{B}$-linear in the first variable and right $\mathcal{B}$ linear in the second variable and satisfies

$$
\eta(x f, y)=\eta(x, f y)
$$

for all $x$ and $y$ in $\Gamma_{0}$ and $f$ in $\mathcal{B}$, there exists a unique homomorphism

$$
\gamma: \Gamma_{0} \otimes_{\mathcal{B}} \Gamma_{0} \longrightarrow N
$$

of $\mathcal{B}$-bimodule such that:

$$
\eta=\gamma \circ \phi
$$

The solution is unique up to isomorphisms. A construction may be found in [4].

As above we can show that $\Gamma_{1}$ has a $\mathcal{B}$-bimodule structure. It is a fact that $\varphi$ is bilinear with respect to the left-module structure in the first variable and the rightmodule structure in the second variable and satisfies

$$
\varphi(x f, y)=\varphi(x, f y)
$$

for all $x$ and $y$ in $\Gamma_{0}$ and $f$ in $\mathcal{B}$. So there exists a unique $\mathcal{B}$-bimodule homomorphism $\gamma$ from $\Gamma_{0} \otimes_{\mathcal{B}} \Gamma_{0}$ to $\Gamma_{1}$ such that

$$
\varphi=\gamma \circ \phi
$$

Now $\gamma$ is defined by

$$
\gamma:\left(\omega_{1} \otimes m_{1}\right) \otimes\left(\omega_{2} \otimes m_{2}\right) \longmapsto\left(\omega_{1} \otimes \omega_{2}\right) \otimes m_{1} m_{2}
$$

The map $\delta$ from $\Gamma_{1}$ to $\Gamma_{0} \otimes_{\mathcal{B}} \Gamma_{0}$ that sends $\left(\omega_{1} \otimes \omega_{2} \otimes m\right)$ to $\left(\omega_{1} \otimes m\right) \otimes\left(\omega_{2} \otimes 1\right)$ is a $\mathcal{B}$-bimodule homomorphism and is the inverse of $\gamma$.

Finally $\left(\Gamma_{1}, \varphi\right)$ is a solution to our universal problem. 
From the preceding proposition, $\Gamma_{0} \otimes_{\mathcal{B}} \Gamma_{0}$ is isomorphic to the space of $\mathcal{C T}(D)$-valued rank 2 tensors. If $\eta_{1}$ and $\eta_{2}$ belong to $\Gamma\left(T^{*} D\right)$ and $s_{1}$ and $s_{2}$ belong to $\mathcal{B}$ then $\left(\eta_{1} \otimes s_{1}\right) \otimes\left(\eta_{2} \otimes s_{2}\right)$ may be identified with the $\mathcal{C} \mathcal{T}(D)$-valued rank 2 tensor that maps $(X \otimes Y)$ to $\eta_{1}(X) \eta_{2}(Y) s_{1} s_{2}$.

We denote

$$
\left(\eta_{1} \otimes s_{1}\right)\left(\eta_{2} \otimes s_{2}\right)=\left(\eta_{1} \eta_{2}\right) \otimes\left(s_{1} s_{2}\right)
$$

the symmetric product of $\left(\eta_{1} \otimes s_{1}\right)$ and $\left(\eta_{2} \otimes s_{2}\right)$. We extend it by linearity. This symmetric product can be identified with the $\mathcal{C} \mathcal{T}(D)$-valued symmetric tensor of rank 2 defined by

$$
(X \otimes Y) \longmapsto \frac{1}{2}\left(\eta_{1}(X) \eta_{2}(Y)+\eta_{2}(X) \eta_{1}(Y)\right) s_{1} s_{2}
$$

Finally we denote $\left(\eta_{1} \otimes s_{1}\right) \underline{\otimes}\left(\eta_{2} \otimes s_{2}\right)$ the element $\left(\eta_{1} \otimes\right.$ $\left.s_{1}\right) \otimes\left(\eta_{2} \otimes s_{2}\right)$ of $\Gamma_{0} \otimes_{\mathcal{B}} \Gamma_{0}$ to emphasize the fact that the tensor product is relative to $\mathcal{B}$.

Acknowledgements The authors thank the "Communauté d'agglomération de La Rochelle" and the European Social Fund (ESF) for financial support. They also thank the reviewers for helpful remarks and suggestions.

\section{References}

1. Atiyah, M. F., MacDonald, I. G.: Introduction to Commutative Algebra. The Advanced Book Program, Perseous Books Reading, Massachusetts (1969)

2. Batard, T., Saint-Jean, C., Berthier, M.: nD images as Clifford bundle sections - Application to segmentation. selected talk to 8th International Conference on Clifford Algebras and their Applications in Mathematical Physics, to be submitted to the proceedings

3. Baylis, W. E. (Editor): Clifford (Geometric) Algebras with Applications to Physics, Mathematics, and Engineering. Birkhauser (1999)

4. Bourbaki, N.: Eléments de mathématiques. Algèbre. Chapitres 1 à 3. Diffusion C.C.L.S Paris (1970)

5. Carron, T.: Segmentation d'images couleur dans la base teinte-luminance-saturation: approche numérique et symbolique. Thèse de doctorat, Université de Savoie (1995)

6. Carron, T., Lambert, P.: Color edge detector using jointly hue, saturation and intensity. IEEE International Conference on Image Processing ICIP, Volume 3, 977-981 (1994)

7. Chevalley, C.: The Algebraic Theory of Spinors and Clifford Algebras. Springer (new edition 1995)

8. Clifford, W. K.: Applications of Grassmann's Extensive Algebra. American Journal of Mathematics, I:350-358 (1878)

9. Cumani, A.: Edge detection in multispectral images. Computer Vision, Graphics, and Image Processing: Graphical Models and Image Processing 53(1), 40-51 (1991)

10. Di Zenzo, S.: A note on the gradient of a multi-image. Computer Vision, Graphics, and Image Processing 33(1), 116-125 (1986)

11. Doubrovine, B., Novikov, S., Fomenko, A.: Géométrie contemporaine, Méthodes et applications, première partie (Géométrie des surfaces, des groupes de transformations et des champs). Editions Mir, Moscou (1982)
12. Evans, C., Ell, T., Sangwine, S.: Colour-sensitive edge detection using hypercomplex filters. In: Proceedings EUSIPCO 2000, pp. 107-110 (2000)

13. Fairchild, M.: Color Appearance Models. John Wiley and Sons, New York (2005)

14. Frankel, T.: The Geometry of Physics: an Introduction. Cambridge University Press (revised edition), 2001

15. Gevers, T., Stokman, H.M.G.: Classifying color transitions into shadow-geometry, illumination, highlight or material edges. In: Proceedings of the International Conference on Image Processing (ICIP), Volume I, 521-524 (2000)

16. Gevers, T., Smeulders, A.W.M.: Color based image segmentation. Pattern Recognition 32, 453-464 (1999)

17. Greub, W., Halperin, S., Vanstone, R.: Connections, Curvature and Cohomology, vol. I-III. Academic Press, New York (1972, 1973 and 1976)

18. Helgason, S.: Differential geometry, Lie groups, and symmetric spaces. Academic Press, London (1978)

19. Hestenes, D.: New Fundations for Classical Mechanics. Fundamental Theories of Physics, Kluwer Academic Publishers (second edition)

20. Hestenes, D.: Space Time Algebra. Gordon and Breach (1966)

21. Hestenes, D., Sobczyk, G.: Clifford algebra to geometric calculus. D. Reidel, Dordrecht (1984)

22. Lenz, R.: Color edge detectors for conical color spaces. In: Proceedings of CGIP 2000. First International Conference on Color in Graphics and Image Processing, pp. 284-289 (2000)

23. Lounesto, P.: Clifford Algebras and Spinors. London Mathematical Society Lecture Notes Series, Cambridge University Press (1997)

24. Postnikov, M.: Leçons de Géométrie. Groupes et algèbres de Lie. Editions Mir, Moscou (1982)

25. Sangwine, S., Ell, T.: Colour image filters based on hypercomplex convolution. IEEE Proceedings-Vision, Image and Signal Processing 147(2), 89-93 (2000)

26. Sapiro, G.: Color snakes. Computer Vision and Image understanding 68(2), 407-416 (1997)

27. Sochen, N., Kimmel, R., Malladi, R.: A general framework for low level vision. IEEE Transactions on Image Processing 7(3), 310-318 (1998)

28. Sommer, G.: Geometric computing with Clifford Algebras. Theorical Foundations and Applications in Computer Vision and Robotics. Springer-Verlag, Berlin (2001)

29. Yezzi, A. jr.: Modified curvature motion for image smothing and enhancement. IEEE Transactions on Image Processing 7(3), 345-352 (1998) 